Research Letter

\title{
Effect of D-amphetamine on dopaminergic neurons of substantia nigra and expression of tyrosine hydroxylase in striatum and pre-frontal cortex of D-amphetamine treated Wistar rats
}

\author{
Sarun Koirala, Sandip Shah, Laxman Khanal \\ BP Koirala Institute of Health Sciences, Dharan, Nepal
}

Received 16 July 2014, Accepted 25 August 2014

(C) 2014, Koirala S., Shah S., Khanal L.

(C) 2014, Russian Open Medical Journal

Abstract: Dopaminergic neurons of the midbrain are the main source of dopamine in the mammalian central nervous system. Dopamine is a chemical messenger active in mesolimbic and mesocortical reward pathways. Dopamine is manufactured in nerve cell bodies in the ventral tegmental area (VTA) and is released in the nucleus accumbens and the prefrontal cortex. The objective was to compare dopaminergic neurons of substantia nigra and level of tyrosine hydroxylase (TH) in the striatum and pre-frontal cortex of D-amphetamine treated wistar mice. 15 Wistar rats were injected subcutaneously with amphetamine $10 \mathrm{mg} / \mathrm{kg}$ body weight till 7 days while the controls groups (15 wistar rats) were injected with normal saline in the same dose. One way-ANOVA test and Post-hoc tests was applied and decreased level of tyrosine hydroxylase was present in striatum (65\%) and pre-frontal cortex (71\%). The percentage of tyrosine hydroxylase in all these areas were highly significant $(p<0.001)$. D-AMPH affects the neuronal cell morphology and decreases expression level of Tyrosine hydroxylase. Degeneration of dopaminergic neurons and damaged synaptic connection in substantia nigra were observed leading to a reduction of stratial dopamine levels.

Keywords: D-amphetamine, tyrosine hydroxylase, substantia nigra, dopamine

Cite as Koirala S, Shah S, Khanal L. Effect of D-amphetamine on dopaminergic neurons of substantia nigra and expression of tyrosine hydroxylase in striatum, pre-frontal cortex of D-amphetamine treated Wistar rats. Russian Open Medical Journal 2014; 3: 0401.

Correspondence to Dr Sarun Koirala. Phone: 97798420 53985. Email: poksun@rediffmail.com

\section{Introduction}

Dopaminergic neurons of the midbrain are the main source of dopamine (DA) in the mammalian central nervous system. Dopamine is a chemical messanger active in Mesolimbic and Mesocortical reward pathways [1]. Dopamine is manufactured in nerve cell bodies in the Ventral Tegmental area (VTA) and is released in the Striatum, Nucleus Accumbens and the Prefrontal Cortex. Motor functions are linked within cell bodies in the substantia nigra which manufacture and release dopamine into the striatum. In the brain, dopamine plays an important role in the regulation of reward and movement [2]. As tyrosine hydroxylase catalyzes the formation of L-DOPA, the rate-limiting step in the biosynthesis of dopamine, tyrosine hydroxylase-deficiency causes important clinical symptoms. Several important diseases of the nervous system are associated with dysfunctions of the dopamine system [3]. The objective of this study was to compare dopaminergic neurons of Substantia Nigra and level of tyrosine hydroxylase (TH) in the striatum, pre-frontal cortex (PFC) and nucleus accumbens (NAC) of D-amphetamine(D-AMPH) treated Wistar rats.

\section{Material and Methods}

Experimental groups of 6 Wistar rats were injected subcutaneously with amphetamine $10 \mathrm{mg} / \mathrm{kg}$ body weight daily for 7 days while the controls groups (6 Wistar rats) were injected with normal saline in the same dose. On day 7 both the groups were deeply anesthetized with $(40 \mathrm{mg} / \mathrm{kg}$ i.p.) sodium pentobarbital. Tissue perfusion and fixation was done transcardially with $4 \%$ paraformaldehyde in 0.1 sodium phosphate buffer (PBS), PH 7.4. Frozen coronal brain sections ( $25 \mu \mathrm{m}$ thick) containing the Substantia Nigra and the Ventral Tegmental Area (VTA) was done with cryostat followed by Immunohistochemical (IHC) stainings and visualized under light microscope. Homogenized Nigral tissue in RIPA (Radio-ImmunoPrecipitation Assay) buffer was centrifuged to remove debris and the supernatants were collected and run to sodium dodecyl sulphatepolyacrylamide gel electrophoresis (SDS-PAGE). The protein bands were transfered to nitrocellulose membrane. The band was quantified by measuring the density of each band using densitometer with scion imaging program.

One way-ANOVA test and Post-hoc tests was applied to see the level of significance. All the data were normalized to the levels of actin expression in the same sample.

\section{Results (Figures 1 and 2)}

Degeneration of dopaminergic neurons and damaged synaptic connection in substantia nigra, was observed leading to reduction of stratial dopamine levels in amphetamine treated group. Decreased level of tyrosine hydroxylase was present in striatum (65.6\%), nucleus accumbens (55.0\%) and pre-frontal cortex (71.8\%). The expression of percentage of tyrosine hydroxylase in all these 3 areas were highly significant $(p<0.001)$ also the mean difference of Tyrosine hydroxylase between Striatum, Nucleus 
accumbens and Prefrontal cortex was reduced $(\mathrm{p}<0.001)$ (PostHoc Test). D-AMPH affects the neuronal cell morphology and decreases expression level of $\mathrm{TH}$.

\section{Discussion}

A consistent abnormality in Parkinson's disease is degeneration of dopaminergic neurons in the Substantia nigra (SN), leading to a reduction of stratial dopamine levels was seen in study conducted by J. Haavik et al. [4]. Parkinson's disease, an age-related degenerative condition causing tremor and motor impairment is caused by loss of dopamine-secreting neurons in the substantia nigra. Our study shows there is decreased level of dopaminic activities in the striatum (65.60 \pm 0.59$)$ in the experimental group. D. Nutt et al. and M. Hooks et al. [5-6] study reveals that schizophrenia has been shown to involve elevated levels of dopamine activity in the mesolimbic pathway and decreased levels of dopamine in the prefrontal cortex. Similarly in a study conducted by Goldman Rakic et al. [7] shows that in schizophrenia, there are elevated levels of dopamine activity in the mesolimbic pathway and decreased levels of dopamine in the Prefrontal cortex. Another study of K.L. Davis [8] shows decreased dopamine activity in attention deficit hyperactivity disorder (ADHD). Study from K. Larsen et al. [9] shows significantly reduced activity of tyrosine hydroxylase in the brains of patients with Alzheimer's disease as compared to healthy individuals. In our study there is decreased level of expression of Tyrosine hydroxylase and dopamine activities $(71.77 \pm 0.58)$ in pre-frontal cortex in the experimental group as compared to other region of control group.

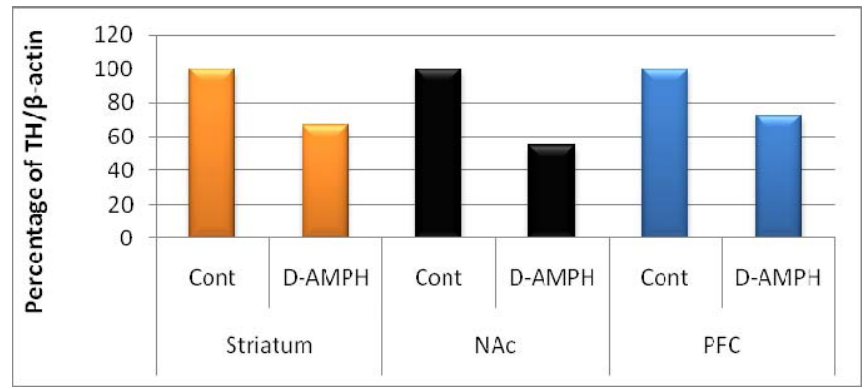

Figure 1. Percentage of expression of Tyrosine hydroxylase in Striatum, Nucleus accumbens and Prefrontal cortex.
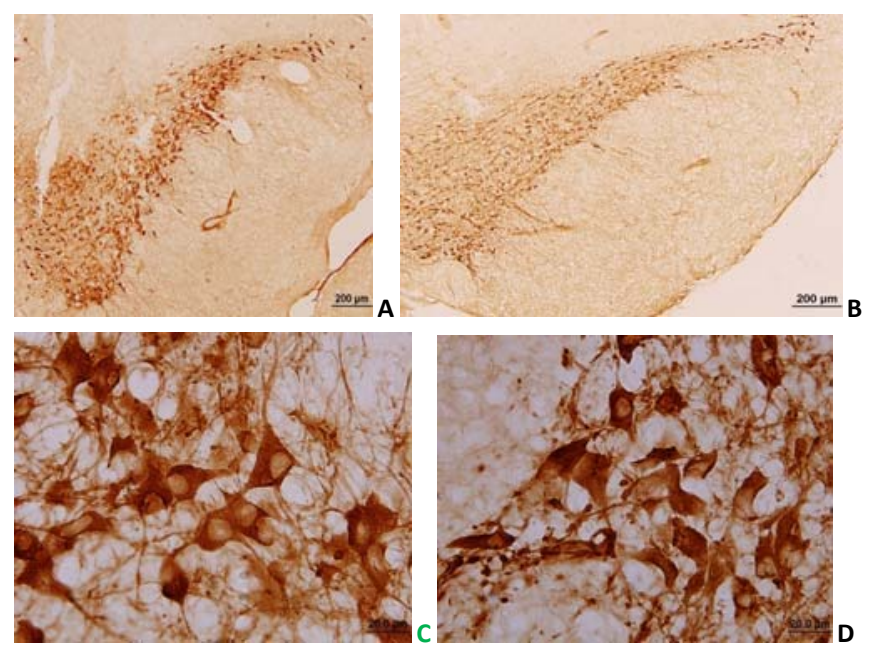

Figure 2. ( $A$ and $C$ ) - Dopaminergic neurons in substantia nigra (Midbrain) of control group; (B and D) - Dopaminergic neurons in substantia nigra of D-AMPH treated group.

\section{Conclusion}

D-AMPH affects the neuronal cell morphology and decreases expression level of TH. Degeneration of dopaminergic neurons and damaged synaptic connection in substantia nigra, was observed leading to a reduction of stratial dopamine levels. Animal models of Parkinson's disease, Schizophrenia, Alzheimer's diseases, attention deficit hyperactivity disorder (ADHD) can be designed for the further clinical experiments

\section{Acknowledgments}

To all the lab members who supported during the research activities.

Conflict of interest: none declared.

\section{References}

1. Schultz W. Multiple dopamine functions at different time courses. Annu Rev Neurosci 2007; 30: 259-288. (PMID: 17600522) (doi: 10.1146/annurev.neuro.28.061604.135722)

2. Arias-Carrión O, Pöppel E. Dopamine, learning and reward-seeking behavior. Act Neurobiol Exp 2007; 67(4): 481-488. (PMID: 18320725)

3. Nagatsu T. Tyrosine hydroxylase: human isoforms, structure and regulation in physiology and pathology. Essays Biochem 1995. 30: 1535. (PMID: 8822146)

4. Haavik J, Toska K. Tyrosine hydroxylase and Parkinson's disease. Molecular Neurobiology 1998; 16(3): 285-309. (PMID: 9626667)

5. Nutt D, King LA, Saulsbury W, Blakemore C. Development of a rational scale to assess the harm of drugs of potential misuse. Lancet 2007; 369: 1047-1053. (doi:10.1016/S0140-6736(07)60464-4)

6. Hooks MS, Jones GH, Neill DB, Justice JB Jr. Individual differences in amphetamine sensitization: dose-dependent effects. Pharmacol Biochem Behav 1992; 41(1): 203-210. (doi: 10.1016/00913057(92)90083-R) (PMID: 153907)

7. Goldman-Rakic PS, Castner SA, Svensson TH, Siever L, Williams GV. Targeting the dopamine D1 receptor in schizophrenia: insights for cognitive dysfunction. Psychopharmacology (Berl) 2004; 174(1): 3-16. (PMID: 15118803) (doi: 10.1007/s00213-004-1793-y)

8. Davis KL, Kahn RS, Ko G, Davidson M. Dopamine in schizophrenia: a review and reconceptualization. American Journal of Psychiatry 1991; 148: 1474-1486. (PMID: 1681750)

9. Larsen K, Fon E, Hastings T, Edwards R, Sulzer D. Methamphetamineinduced degeneration of dopaminergic neurons involves autophagy and upregulation of dopamine synthesis. J Neurosci 2002; 22(20): 8951-8960. (PMID: 12388602)

\section{Authors:}

Sarun Koirala - MS, Assistant Professor, Department of Human Anatomy, BP Koirala Institute of Health Sciences, Dharan, Nepal;

Sandip Shah - MS, Assistant Professor, Department of Human Anatomy, BP Koirala Institute of Health Sciences, Dharan, Nepal;

Laxman Khanal - MS, Resident, Department of Human Anatomy, BP Koirala Institute of Health Sciences, Dharan, Nepal. 